\title{
Rotating Topological Black Brnaes in Various Dimensions and AdS/CFT Correspondence
}

\author{
M. H. Dehghani \\ Physics Department and Biruni Observatory, Shiraz University, Shiraz 71454, Iran \\ We consider rotating topological black branes with one rotational parameter in \\ various dimensions. Also a general five-dimensional higher genus solution of the \\ Einstein equation with a negative cosmological constant which represents a topolog- \\ ical black brane with two rotational parameters is introduced. We find out that the \\ counterterms inspired by conformal field theory introduced by Kraus, Larsen and \\ Sieblink cannot remove the divergences in $r$ of the action in more than five dimen- \\ sions. We modify the counterterms by adding a curvature invariant term to it. Using \\ the modified counterterms we show that the $r$ divergences of the action, the mass, \\ and the angular momentum densities of these spacetimes are removed. We also find \\ out in the limit of $m=0$ the mass density of these spacetimes in odd dimensions is \\ not zero.
}

\section{INTRODUCTION}

The AdS conformal field theory (CFT) correspondence conjecture asserts that there is a relation between a super gravity or string theory in $(n+1)$-dimensional anti-de Sitter (AdS) spacetimes and a conformal field theory (CFT) living on an $n$-dimensional boundary [1]. This equivalence enables one to remove the divergences of the action and conserved quantities of gravity in the same way as one does in field theory. Indeed the CFT-inspired boundary counterterms furnish a means for calculating the action and conserved charges intrinsically without reliance on any reference spacetime [2, 3, 4]. The efficiency of this approach has been demonstrated in a broad range of examples in the spacially infinite limit, where the AdS/CFT correspondence applies [5, 6, 7]. Although the AdS/CFT correspondence applies for the case of spacially infinite boundary, it was also employed for the computation of the conserved and thermodynamical quantities in the case of a finite boundary [8]. These ideas have been recently extended to the case of asymptotically de Sitter spacetimes [9, 10]. 
In this paper we want to apply the AdS/CFT correspondence to the case of rotating topological black branes whose $(n-1)$-surfaces at fixed $r$ and $t$ have nontrivial topology with nonconstant curvature [11, 12]. Although in the non-rotating case one can compactify these hypersurfaces to obtain topological black holes [13, 14], in the rotating case this cannot be done. The AdS/CFT correspondence has been used for the case of topological black holes with constant curvature horizon [5, [5, [16], but it has not applied to the case of rotating higher genus black branes until now. Thus it is worthwhile to investigate the efficiency of the AdS/CFT correspondence for this type of black branes. It is remarkable to mention that the counterterms introduced in Ref. [2] cannot remove the $r$ divergences in the action of rotating topological black branes in more than five dimensions. But as it is known the expression for $I_{c t}$ obtained by the algorithm given in Ref. [2] is not unique, and one can add any functional of curvature invariants that vanish at infinity in a given dimension. So, we first modify the counterterms and then we show that the $r$ divergences of the action and the conserved charge densities of these spacetimes in various dimension up to seven are removed. Also we introduce the general five-dimensional topological Kerr-AdS solution which describes rotating black brane in AdS spacetime with two rotational parameters.

The outline of our paper is as follows. In Sec. II we review the basic formalism and introduce the modified counterterms. Section [IT] will be devoted to the consideration of topological Kerr-AdS metric with one rotational parameter in various dimensions. We also show that the modified counterterms remove the divergences in $r$ for the densities of the action and the conserved charges. In Sec. IV the general five-dimensional topological KerrAdS spacetimes with two rotational parameters is introduced, and the action and conserved charge densities computed. We finish our paper with some concluding remarks.

\section{THE ACTION AND CONSERVED QUANTITIES}

The gravitational action of $(n+1)$-dimensional spacetimes $\mathcal{M}$, with boundary $\delta \mathcal{M}$ is

$$
I_{G}=-\frac{1}{16 \pi} \int_{\mathcal{M}} d^{n+1} x \sqrt{-g}(\mathcal{R}-2 \Lambda)+\frac{1}{8 \pi} \int_{\partial \mathcal{M}} d^{n} x \sqrt{-\gamma} \Theta(\gamma) .
$$

The first term is the Einstein-Hilbert volume (or bulk) term with negative cosmological constant $\Lambda=-n(n-1) /\left(2 l^{2}\right)$ and the second term is the Gibbons-Hawking boundary term which is chosen such that the variational principle is well-defined. The manifold $\mathcal{M}$ 
has metric $g_{\mu \nu}$ and covariant derivative $\nabla_{\mu}$. $\Theta$ is the trace of the extrinsic curvature $\Theta^{\mu \nu}$ of any boundary(ies) $\partial \mathcal{M}$ of the manifold $\mathcal{M}$, with induced metric(s) $\gamma_{i j}$. In general the first and second terms of Eq. (1) are both divergent when evaluated on solutions, as is the Hamiltonian, and other associated conserved quantities. Rather than eliminating these divergences by incorporating a reference term in the spacetime [17, 18], a new term, $I_{c t}$, is added to the action which is a functional only of the boundary curvature invariants. For an asymptotically AdS spacetime, this has been done in Ref. [2] through the use of an algorithmic procedure. These counterterms up to seven dimensions are

$I_{c t}=\frac{1}{8 \pi} \int_{\partial \mathcal{M}_{\infty}} d^{n} x \sqrt{-\gamma}\left\{-\frac{n-1}{l}-\frac{l R}{2(n-2)}-\frac{l^{3}}{2(n-4)(n-2)^{2}}\left(R_{a b} R^{a b}-\frac{n}{4(n-1)} R^{2}\right)+\ldots\right\}$,

where $R, R_{a b c d}$, and $R_{a b}$ are the Ricci scalar, Riemann tensor and Ricci tensor of the boundary metric $\gamma_{a b}$. Indeed, there may exist a very large number of possible invariants one could add in a given dimension, but in Ref. [2] only a finite number of them have been introduced. These counterterms have been used by many authors for a wide variety of the spacetimes, including Schwarzschild-AdS, topological Schwarzschild-AdS, Kerr-AdS, Taub-NUT-AdS, Taub-bolt-AdS, and Taub-bolt-Kerr-AdS [5, 6, 7]. Although the counterterms introduced in Ref. [2] can remove the divergences of the action and conserved charges of these spacetimes, one may show that these counterterms cannot remove the divergences in $r$ of the action of topological Kerr-AdS in more than five dimensions. In order to remove the $r$ divergences, we should modify the counterterms (22) by adding the following curvature invariant term to it:

$$
I_{c t}^{\prime}=\frac{1}{8 \pi} \int_{\partial \mathcal{M}_{\infty}} d^{n} x \sqrt{-\gamma} \frac{l^{3}}{2(n-1)(n-4)\left(n^{2}-4\right)} \nabla^{2} R .
$$

It is remarkable to mention that the counterterm (3) is equal to zero for the case of black holes whose horizons have positive or negative constant curvature. The total action can be written as a linear combination of the gravity term (国) and the counterterms (2) and (河) as

$$
I=I_{G}+I_{c t}+I_{c t}^{\prime}
$$

Using the Brown and York definition [17] one can construct a divergence free stress-energy tensor from the total action (四) as

$$
T^{a b}=\frac{1}{8 \pi}\left\{\left(\Theta^{a b}-\Theta \gamma^{a b}\right)-\frac{n-1}{l} \gamma^{a b}+\frac{l}{n-2}\left(R^{a b}-\frac{1}{2} R \gamma^{a b}\right)\right.
$$




$$
\begin{aligned}
& +\frac{l^{3}}{(n-4)(n-2)^{2}}\left[-\frac{1}{2} \gamma^{a b}\left(R^{c d} R_{c d}-\frac{n}{4(n-1)} R^{2}\right)-\frac{n}{2(n-2)} R R^{a b}\right. \\
& \left.\left.+2 R_{c d} R^{a c b d}-\frac{n-2}{2(n-1)} \nabla^{a} \nabla^{b} R+\nabla^{2} R^{a b}-\frac{1}{2(n-1)} \gamma^{a b} \nabla^{2} R\right]+\ldots\right\} .
\end{aligned}
$$

To compute the conserved charges of the spacetime, one should choose a spacelike surface $\mathcal{B}$ in $\partial \mathcal{M}$ with metric $\sigma_{i j}$, and write the boundary metric in ADM form:

$$
\gamma_{a b} d x^{a} d x^{a}=-N^{2} d t^{2}+\sigma_{i j}\left(d \phi^{i}+V^{i} d t\right)\left(d \phi^{j}+V^{j} d t\right)
$$

where the coordinates $\phi^{i}$ are the angular variables parametrizing the hypersurface of constant $r$ around the origin. Then the conserved quantities associated with the stress tensors of Eq. (5) can be written as

$$
\mathcal{Q}(\xi)=\int_{\mathcal{B}_{\infty}} d^{n-1} \phi \sqrt{\sigma} T_{a b} n^{a} \xi^{b}
$$

where $\sigma$ is the determinant of the metric $\sigma_{i j}, \xi$ and $n^{a}$ are the Killing vector field and the unit normal vector on the boundary $\mathcal{B}$.

Now in the following sections we study the implications of including the modified counterterms for the class of rotating topological spacetimes in various dimensions. For each Killing vector $\xi$, there exist an associated conserved charge. For our case, rotating topological spacetimes, the first Killing vector is $\xi=\partial / \partial t$ and therefore its associated conserved charge is the total mass of the system enclosed by the boundary given by

$$
M=\int_{\mathcal{B}_{\infty}} d^{n-1} \phi \sqrt{\sigma} T_{a b} n^{a} \xi^{b} .
$$

The charge associated to a rotational Killing symmetry generated by $\zeta=\partial / \partial \phi$ is the angular momentum written as

$$
J=\int_{\mathcal{B}_{\infty}} d^{n-1} \phi \sqrt{\sigma} T_{a b} n^{a} \zeta^{b}
$$

but in our case there is no global rotational Killing symmetry. However, the vector $\zeta=\partial / \partial \phi$, although it is not a Killing vector, obeys locally the condition $\nabla_{(a} \zeta_{b)}=0$ and is therefore a kind of approximate symmetry, or a locally exact symmetry.

In the context of the AdS/CFT correspondence the limit in which the boundary $\mathcal{B}$ becomes infinite $\left(\mathcal{B}_{\infty}\right)$ is taken, and the counterterm prescription ensures that the divergences in $r$ of the action, mass, and angular momentum are removed. No embedding of the surface $\mathcal{B}$ into a reference spacetime is required and the quantities which are computed are intrinsic to the spacetimes. In our case since the boundary $\mathcal{B}$ cannot be compactified, the divergences in the angular variables parametrizing the hypersurface $\mathcal{B}$ remain. 


\section{TOPOLOGICAL KERR-ADS METRIC WITH ONE ROTATIONAL PARAMETER}

In this section we consider the class of higher genus $(g>1)$ rotating topological solutions of the Einstein equation with negative cosmological constant in various dimensions with one rotational parameter. These solutions in $(n+1)$ dimension can be written as [11, 12]:

$$
\begin{aligned}
d s^{2}= & -\frac{\Delta_{r}}{\rho^{2}}\left(d t+\frac{a}{\Xi} \sinh ^{2} \chi d \phi\right)^{2}+\frac{\rho^{2}}{\Delta_{r}} d r^{2}+\frac{\rho^{2}}{\Delta_{\chi}} d \chi^{2} \\
& +\frac{\Delta_{\chi} \sinh ^{2} \chi}{\rho^{2}}\left[a d t-\frac{\left(r^{2}+a^{2}\right)}{\Xi} d \phi\right]^{2}+r^{2} \cosh ^{2} \chi d \Sigma_{n-3},
\end{aligned}
$$

where $d \Sigma_{n-3}$ is the metric of a unit $(n-3)$-dimensional compact pseudosphere and

$$
\begin{aligned}
\Delta_{r} & =\left(r^{2}+a^{2}\right)\left(\frac{r^{2}}{l^{2}}-1\right)-2 m r^{4-n}, \\
\Delta_{\chi} & =1+\frac{a^{2}}{l^{2}} \cosh ^{2} \chi \\
\Xi & =1+\frac{a^{2}}{l^{2}} \\
\rho^{2} & =r^{2}+a^{2} \cosh ^{2} \chi .
\end{aligned}
$$

One may note that Eqs. (9 and 10) describe spacetimes which reduce in the case of $a=0$, to the topological Schwarzschild -AdS black holes in $(n+1)$ dimension. Hence we expect the parameters $m$ and $a$ to be associated with the mass and angular momentum of the spacetime respectively. The metric of Eq. (9) is a limit case of the generalized Petrov-type D solution of the Einstein equation with cosmological constant in $(n+1)$ dimension introduced in [12.

The metric induced on a spacelike $(n-1)$-surface at fixed coordinates $r$ and $t$ is

$$
\frac{\rho^{2}}{\Delta_{\chi}} d \chi^{2}+\left[\left(r^{2}+a^{2}\right)^{2} \Delta_{\chi}-a^{2} \sinh ^{2} \chi \Delta_{r}\right] \frac{\sinh ^{2} \chi}{\rho^{2}} d \phi^{2}+r^{2} d \Sigma_{n-3} .
$$

Note that the Gaussian curvature of this metric is not constant and one cannot compactify the $(\chi-\phi)$ sector [12]. In order to have a Euclidean metric we have to require that the term in the bracket of Eq. (11) be positive. That is

$$
\left(r^{2}+a^{2}\right)^{2} \Delta_{\chi}-a^{2} \sinh ^{2} \chi \Delta_{r}>0 .
$$

This condition restricts the allowed values of the mass parameter and we will consider it in various dimensions in the following sections. 


\section{A. Topological Kerr-AdS 4 Metric}

The metric of Eqs. (9) and (10) for $n=3$ is a limit case of the metric of Plebanski and Demianski 19 which is the most general known Petrov type-D solution of the source-free Einstein-Maxwell equation with cosmological constant in four dimensions [11]. This metric has two inner and outer horizons located at $r_{-}$and $r_{+}$provided the mass parameter $m$ lies between $m_{1, \text { crit }} \leq m \leq m_{2, \text { crit }}$ and only an outer horizon if the parameter $m$ is outside of this range, where $m_{1, c r i t}$ and $m_{2, \text { crit }}$ are the two critical masses given by:

$$
\begin{aligned}
& m_{1, \text { crit }} \equiv-\frac{l}{3 \sqrt{6}} \sqrt{1+33 \frac{a^{2}}{l^{2}}\left(1-\frac{a^{2}}{l^{2}}\right)-\frac{a^{6}}{l^{6}}+\left(1-14 \frac{a^{2}}{l^{2}}+\frac{a^{4}}{l^{4}}\right)^{3 / 2}}, \\
& m_{2, \text { crit }} \equiv-\frac{l}{3 \sqrt{6}} \sqrt{1+33 \frac{a^{2}}{l^{2}}\left(1-\frac{a^{2}}{l^{2}}\right)-\frac{a^{6}}{l^{6}}-\left(1-14 \frac{a^{2}}{l^{2}}+\frac{a^{4}}{l^{4}}\right)^{3 / 2}} .
\end{aligned}
$$

The metric induced on the 2-hypersurface at fixed $r=$ const $>r_{+}$and $t=$ const is Euclidean provided the parameter $m \geq m_{1, \text { crit }}$. Thus although this metric has a horizon for all values of $m$, to have a Euclidean metric on the 2-surface outside the horizon we should restrict the allowed values of the mass parameter to $m \geq m_{1, c r i t}$. Note that this range introduced here is a little greater than the one introduced in Ref. [11] and this is due to the fact that we restrict ourself to outside of the horizon.

Using Eq. (4) the action in the limit in which the boundary becomes infinite can be written as

$$
I_{4}=\beta_{+}^{(4)} \int_{\mathcal{B}_{\infty}} \mathcal{I}_{B}^{(4)} \sinh \chi d \chi d \phi
$$

where $\beta_{+}^{(4)}$ and $\mathcal{I}_{B}^{(4)}$ are the inverse Hawking temperature of the event horizon and the action density on the boundary at infinity given as

$$
\begin{aligned}
\beta_{+}^{(4)} & =\frac{4 \pi l^{2} r_{+}\left(r_{+}^{2}+a^{2}\right)}{3 r_{+}^{4}+\left(a^{2}-l^{2}\right) r_{+}^{2}+a^{2} l^{2}} \\
\mathcal{I}_{B}^{(4)} & =-\frac{1}{8 \pi \Xi}\left\{m-r_{+}\left(\frac{r_{+}^{2}}{l^{2}}+3 \frac{a^{2}}{l^{2}} \cosh ^{2} \chi\right)\right\}
\end{aligned}
$$

Note that the $r$ divergences of the action density $\mathcal{I}_{B}^{(4)}$ on the boundary at infinity are removed, but since one cannot compactify $\mathcal{B}$, the divergences in $\chi$ still remain [12]. Using Eqs. (7) and (8) the total mass $M$ and the total angular momentum $J_{\phi}$ are

$$
M=\int_{\mathcal{B}_{\infty}} \mathcal{M}_{B}^{(4)} \sinh \chi d \chi d \phi ; \quad \mathcal{M}_{B}^{(4)}=-\frac{1}{4 \pi} \frac{m}{\Xi}
$$




$$
J_{\phi}=\int_{\mathcal{B}_{\infty}} \mathcal{J}_{B}^{(4)} \sinh \chi d \chi d \phi ; \quad \mathcal{J}_{B}^{(4)}=-\frac{3 m a}{8 \pi \Xi^{2}} \sinh ^{2} \chi
$$

These expressions show that the parameters $m$ and $a$ could be associated with the mass and angular momentum of the spacetime, respectively. As in the case of action, again the $r$ divergences have been removed as one expects from AdS/CFT correspondence.

\section{B. Topological Kerr-AdS Metric $_{5}$}

For the case of $n=4$ the metric given by Eqs. (9) and (10) has two inner and outer horizons located at $r_{-}$and $r_{+}$, provided the parameter $m$ lies between $m_{1, \text { crit }} \leq m \leq m_{2, c r i t}$, and only an outer horizon if the parameter $m>m_{2, \text { crit }}$, where $m_{1, \text { crit }}$ and $m_{2, \text { crit }}$ are the two critical masses given by

$$
\begin{aligned}
& m_{1, c r i t} \equiv-\frac{l^{2}}{8}\left(1+\frac{a^{2}}{l^{2}}\right)^{2}, \\
& m_{2, c r i t} \equiv-\frac{1}{2} a^{2} .
\end{aligned}
$$

The metric induced on the 3-hypersurface at fixed $r=$ const $>r_{+}$and $t=$ const is Euclidean for all the allowed values of the mass parameter $m>m_{1, \text { crit }}$. Using Eq. (4⿴囗十), one can write the total action as

$$
I_{5}=\beta_{+}^{(5)} \int_{\mathcal{B}_{\infty}} \mathcal{I}_{B}^{(5)} \cosh \chi \sinh \chi d \chi d \phi d \psi,
$$

where $\beta_{+}^{(5)}$ and $\mathcal{I}_{B}^{(5)}$ are the inverse Hawking temperature of the event horizon and the action density on the boundary at infinity is given as

$$
\begin{aligned}
& \beta_{+}^{(5)}=\frac{2 \pi l^{2}\left(r_{+}^{2}+a^{2}\right)}{r_{+}\left(2 r_{+}^{2}+a^{2}-l^{2}\right)}, \\
& \mathcal{I}_{B}^{(5)}=-\frac{1}{64 \pi \Xi}\left\{8 m+l^{2}\left[3\left(1-\frac{a^{2}}{l^{2}}\right)^{2}-8 \frac{r_{+}^{4}}{l^{4}}+2\left(9 \frac{a^{2}}{l^{2}}-9 \frac{a^{4}}{l^{4}}-8 \frac{a^{2} r_{+}^{2}}{l^{4}}\right) \cosh ^{2} \chi+19 \frac{a^{4}}{l^{4}} \cosh ^{4} \chi\right]\right\} .
\end{aligned}
$$

Using Eqs. (7) and (8) the total mass $M$ and the total angular momentum $J_{\phi}$ can be written as

$$
\begin{aligned}
M & =\int_{\mathcal{B}_{\infty}} \mathcal{M}_{B}^{(5)} \cosh \chi \sinh \chi d \chi d \phi d \psi, \\
J_{\phi} & =\int_{\mathcal{B}_{\infty}} \mathcal{J}_{B}^{(5)} \cosh \chi \sinh \chi d \chi d \phi d \psi,
\end{aligned}
$$


where the mass and angular momentum densities $\mathcal{M}_{B}^{(5)}$ and $\mathcal{J}_{B}^{(5)}$ are

$$
\begin{aligned}
\mathcal{M}_{B}^{(5)} & =-\frac{1}{64 \pi \Xi}\left\{24 m+l^{2}\left[\left(4-\Xi^{2}\right)+2(6-\Xi) \frac{a^{2}}{l^{2}} \cosh ^{2} \chi+7 \frac{a^{4}}{l^{4}} \cosh ^{4} \chi\right]\right\} \\
\mathcal{J}_{B}^{(5)} & =-\frac{m a}{2 \pi \Xi^{2}} \sinh ^{2} \chi .
\end{aligned}
$$

It is remarkable to note that the mass density $\mathcal{M}_{S}^{(5)}$ computed in Eq. (24) is not zero in the limit of $m=0$. This is a common feature for all the asymptotically (A)dS spacetimes. Again the $r$ divergences of the action, mass, and angular momentum densities are removed but the divergences in $\chi$ still remain.

\section{Topological Kerr-AdS 7 Metric}

The topological Kerr-AdS 7 with one rotational parameter given by Eqs. (9) and (10) has two inner and outer horizons located at $r_{-}$and $r_{+}$provided the mass parameter $m$ lies between $m_{1, \text { crit }} \leq m \leq m_{2, \text { crit }}$, and only an outer horizon if the parameter $m$ is greater than $m_{2, \text { crit }}$ where $m_{1, \text { crit }}$ and $m_{2, \text { crit }}$ are the two critical masses given by

$$
\begin{aligned}
& m_{1, \text { crit }} \equiv-\frac{l^{4}}{27}\left\{1+\frac{3}{2} \frac{a^{2}}{l^{2}}\left(1-\frac{a^{2}}{l^{2}}\right)-\frac{a^{6}}{l^{6}}+\left(1+\frac{a^{2}}{l^{2}}+\frac{a^{4}}{l^{4}}\right)^{3 / 2}\right\}, \\
& m_{2, \text { crit }} \equiv-\frac{l^{4}}{27}\left\{1+\frac{3}{2} \frac{a^{2}}{l^{2}}\left(1-\frac{a^{2}}{l^{2}}\right)-\frac{a^{6}}{l^{6}}-\left(1+\frac{a^{2}}{l^{2}}+\frac{a^{4}}{l^{4}}\right)^{3 / 2}\right\} .
\end{aligned}
$$

The metric induced on the 5-hypersurface at fixed $r=$ const $>r_{+}$and $t=$ const is Euclidean for all the allowed values of the mass parameter $m \geq m_{1, \text { crit }}$. Using Eq. (幽, one can write the total action as

$$
I_{7}=\beta_{+}^{(5)} \int_{\mathcal{B}_{\infty}} \mathcal{I}_{B}^{(7)} \cosh ^{3} \chi \sinh \chi \cosh \psi \sinh \psi d \chi d \phi d \psi d \eta d \zeta
$$

where $\beta_{+}^{(7)}$ and $\mathcal{I}_{B}^{(7)}$ are the inverse Hawking temperature of the event horizon and the action density on the boundary at infinity given as

$$
\begin{aligned}
\beta_{+}^{(7)}= & \frac{2 \pi l^{2} r_{+}\left(r_{+}^{2}+a^{2}\right)}{3 r_{+}^{4}+2 r_{+}^{2}\left(a^{2}-l^{2}\right)-a^{2} l^{2}}, \\
\mathcal{I}_{B}^{(7)}= & -\frac{1}{640 \pi \Xi}\left\{80 m+l^{4}\left[25\left(1-\frac{a^{6}}{l^{6}}\right)-47 \frac{a^{2}}{l^{2}}\left(1-\frac{a^{2}}{l^{2}}\right)-80 \frac{r_{+}^{6}}{l^{6}}\right.\right. \\
& \left.\left.+6\left(18 \Xi^{2}-57 \frac{a^{2}}{l^{2}}-20 \frac{r_{+}^{4}}{l^{4}}\right) \frac{a^{2}}{l^{2}} \cosh ^{2} \chi+79\left(1-\frac{a^{2}}{l^{2}}\right) \frac{a^{4}}{l^{4}} \cosh ^{4}-20 \frac{a^{6}}{l^{6}} \cosh ^{6} \chi\right]\right\} .
\end{aligned}
$$


Again using Eqs. (7 \& 8), the total mass $M$ and the total angular momentum $J_{\phi}$ can be written as:

$$
\begin{aligned}
& M=\int_{\mathcal{B}_{\infty}} \mathcal{M}_{B}^{(7)} \cosh ^{3} \chi \sinh \chi \cosh \psi \sinh \psi d \chi d \phi d \psi d \eta d \zeta ; \\
& J_{\phi}=\int_{\mathcal{B}_{\infty}} \mathcal{J}_{B}^{(7)} \cosh ^{3} \chi \sinh \chi \cosh \psi \sinh \psi d \chi d \phi d \psi d \eta d \zeta
\end{aligned}
$$

where the mass and angular momentum densities are:

$$
\begin{aligned}
\mathcal{M}_{B}^{(7)=} & -\frac{1}{640 \pi \Xi}\left\{400 m+l^{4}\left[25-101 \frac{a^{2}}{l^{2}}-\frac{a^{4}}{l^{4}}+5 \frac{a^{6}}{l^{6}}\right.\right. \\
& \left.\left.+3\left(63-56 \frac{a^{2}}{l^{2}}-\frac{a^{4}}{l^{4}}\right) \frac{a^{2}}{l^{2}} \cosh ^{2} \chi+3(94-17 \Xi) \frac{a^{4}}{l^{4}} \cosh ^{4} \chi+55 \frac{a^{6}}{l^{6}} \cosh ^{6} \chi\right]\right\}, \\
\mathcal{J}_{B}^{(7)}= & -\frac{3}{4 \pi} \frac{m a}{\Xi^{2}} \sinh ^{2} \chi .
\end{aligned}
$$

Although the divergences in $r$ have been removed, the $\chi$ divergences still remain.

\section{THE GENERAL TOPOLOGICAL KERR-ADS METRIC IN FIVE DIMENSION}

The general rotating black hole in five dimensions can have at most two rotational parameters [20]. Thus one can write the metric of five dimensional topological black brane with two rotational parameters as

$$
\begin{aligned}
d s^{2}=-\frac{\Delta_{r}}{\rho^{2}} & \left(d t+\frac{a \sinh ^{2} \theta}{\Xi_{a}} d \phi-\frac{b \cosh ^{2} \theta}{\Xi_{a}} d \psi\right)^{2}-\frac{\rho^{2}}{\Delta_{\theta}} d \theta^{2}+\frac{\rho^{2}}{\Delta_{r}} d r^{2} \\
& +\frac{1+r^{2} / l^{2}}{r^{2} \rho^{2}}\left[a b d t+\frac{b\left(r^{2}+a^{2}\right) \sinh ^{2} \theta}{\Xi_{a}} d \phi-\frac{a\left(r^{2}+b^{2}\right) \cosh ^{2} \theta}{\Xi_{b}} d \psi\right]^{2} \\
& -\frac{\Delta_{\theta} \sinh ^{2} \theta}{\rho^{2}}\left(a d t-\frac{r^{2}+a^{2}}{\Xi_{a}} d \phi\right)+\frac{\Delta_{\theta} \cosh ^{2} \theta}{\rho^{2}}\left(b d t-\frac{r^{2}+b^{2}}{\Xi_{b}} d \phi\right),
\end{aligned}
$$

where

$$
\begin{aligned}
& \Delta_{r}=\frac{1}{r^{2}}\left(r^{2}+a^{2}\right)\left(r^{2}+b^{2}\right)\left(1+\frac{r^{2}}{l^{2}}\right)-2 m, \\
& \Delta_{\theta}=1-\frac{a^{2}}{l^{2}} \cosh ^{2} \theta+\frac{b^{2}}{l^{2}} \sinh ^{2} \theta, \\
& \Xi_{a}=1+\frac{a^{2}}{l^{2}}, \quad \Xi_{b}=1+\frac{b^{2}}{l^{2}}, \\
& \rho^{2}=r^{2}+a^{2} \cosh ^{2} \theta-b^{2} \sinh ^{2} \theta .
\end{aligned}
$$


The metric given by Eqs. (31) and (32) is the solution of the Einstein equation with negative cosmological constant. It has two inner and outer horizons provided the mass parameter $m$ is greater than the critical mass $m_{\text {crit }}$ :

$$
\begin{aligned}
m_{\text {crit }} & =\frac{1}{24 l^{2}}\left\{\Upsilon^{1 / 3}+6^{4} \mu^{2}\left(a^{2} b^{2} l^{2}\right)^{4 / 3}\left(1+\mu^{6}\right) \Upsilon^{-1 / 3}+15 \mu^{4}\left(a^{2} b^{2} l^{2}\right)^{2 / 3}-4\left(a^{4}+b^{4}+l^{4}\right)\right\} \\
\Upsilon & =18^{3} a^{4} b^{4} l^{4}\left\{1+20 \mu^{6}-\mu^{12}+\left(1-8 \mu^{6}\right)^{3 / 2}\right\} \\
\mu^{6} & =\frac{\left(a^{2}+b^{2}+l^{2}\right)^{3}}{216 a^{2} b^{2} l^{2}} .
\end{aligned}
$$

Using Eq. (雨), one can write the total action as

$$
I_{5}^{\prime}=\beta_{+}^{(5)} \int_{\mathcal{B}_{\infty}} \mathcal{I}_{S}^{\prime(5)} \cosh \chi \sinh \chi d \chi d \phi d \psi
$$

where $\beta_{+}^{\prime(5)}$ and $\mathcal{I}_{B}^{\prime(5)}$ are the inverse Hawking temperature of the event horizon and the action density on the boundary at infinity given as

$$
\begin{aligned}
\beta_{+}^{\prime(5)}= & -\frac{2 \pi l^{2} r_{+}\left(r_{+}^{2}+a^{2}\right)\left(r_{+}^{2}+b^{2}\right)}{2 r_{+}^{6}+\left(a^{2}+b^{2}+l^{2}\right) r^{4}+a^{2} b^{2} l^{2}}, \\
\mathcal{I}_{B}^{\prime(5)}=- & \frac{1}{64 \pi \Xi_{a} \Xi_{b}}\left\{8 m+3\left(a^{2}+l^{2}\right)\left(1-4 \frac{b^{2}}{l^{2}}\right)+\frac{4}{l^{2}}\left(b^{4}-b^{2} r_{+}^{2}-2 r_{+}^{4}\right)\right. \\
& \left.-2\left[10 \Xi_{b}-9 \Xi_{a}+8\left(1+\frac{r_{+}^{2}}{l^{2}}\right)\right]\left(a^{2}-b^{2}\right) \cosh ^{2} \chi+\frac{19}{l^{2}}\left(a^{2}-b^{2}\right)^{2} \cosh ^{4} \chi\right\} .
\end{aligned}
$$

Again the total mass $M$ and the total angular momenta $J_{\phi}$ and $J_{\psi}$ calculated from Eqs. (7) and (8) are given by Eqs. (22) and (23) where the mass and angular momentum densities are

$$
\begin{aligned}
\mathcal{M}_{B}^{\prime(5)}=- & \frac{1}{64 \pi \Xi_{a} \Xi_{b}}\left\{24 m+l^{2}\left(4 \Xi_{b}^{2}-\Xi_{a}^{2}\right)\right. \\
& \left.-2\left(6 \Xi_{b}-\Xi_{a}\right)\left(a^{2}-b^{2}\right) \cosh ^{2} \chi+\frac{7}{l^{2}}\left(a^{2}-b^{2}\right)^{2} \cosh ^{4} \chi\right\}, \\
\mathcal{J}_{\phi}^{\prime(5)}=- & \frac{m a}{2 \pi \Xi_{a}^{2} \Xi_{b}} \sinh ^{2} \chi, \quad \mathcal{J}_{\psi}^{\prime(5)}=-\frac{m b}{2 \pi \Xi_{a} \Xi_{b}^{2}} \cosh ^{2} \chi .
\end{aligned}
$$

Again note that the mass density in the limit of $m=0$ vanishes and all the $r$ divergences in the densities are removed.

\section{CLOSING REMARKS}

In this paper we have considered a class of higher genus solutions of the Einstein equation with negative cosmological constant which represents rotating topological black branes with 
one rotating parameter in more than three dimensions. Also we have introduced the general topological Kerr-AdS solutions in five dimensions with two rotating parameters. Since one is interested in outside the horizon we have modified the allowed values of the mass parameter $m$, for topological Kerr-AdS 4 given in Ref. [11], and introduced these values for topological Kerr-AdS in various dimensions up to seven.

The main aim of this paper was the investigation of the efficiency of the AdS/CFT correspondence for these rotating topological black branes. We have found out that the counterterms introduced in [2] cannot remove the $r$ divergences of the action of these spacetimes in more than five dimensions. But as it is well known, the expression for $I_{c t}$ obtained by the algorithm given in ref. [2] is not unique, and one can add any functional of curvature invariants that vanish at infinity in a given dimension. Thus in order to remove the $r$ divergences in action we modified these counterterms by adding a suitable curvature invariant term to it. Although this term vanishes for black holes whose horizons have negative or positive curvature constant, we have shown that it removes the divergences of the action of higher genus rotating topological black branes in more than five dimensions. Using modified counterterms, we have computed the conserved mass and locally conserved angular momentum densities of rotating topological Kerr-AdS spacetimes in various dimensions through the use of a Brown-York boundary stress tensor. We found out that although the $r$ divergences of all the densities on the boundary $\mathcal{B}_{\infty}$ are removed, the divergences in $\chi$ still remain. This is due to the fact that one cannot compactify the boundary $\mathcal{B}$. As in the case of asymptotically (A)dS black holes, we found out that in the limit of $m=0$ the mass density of these spacetimes in odd dimensions is not zero.

The efficiency of the AdS/CFT correspondence for higher dimensional rotating topological black branes and other solutions of the Einstein equation such as cosmological $C$-metric and the extension of these ideas to the case of asymptotically de Sitter spacetimes remain interesting subjects for future investigations. Also removing the divergences in $\chi$ of the action and conserved charges and calculating finite values of these quantities remain an open issue, which we leave for the future.

[1] J. Maldacena, Adv. Theor. Math. Phys. 2, 231 (1998); E. Witten, ibid. 2, 253 (1998); O. Aharony, S. S. Gubser, J. Maldacena, H. Ooguri and Y. Oz, Phys. Rep. 323, 183 (2000). 
[2] P. Kraus, F. Larsen and R. Siebelink, Nucl. Phys. B563, 259 (1999).

[3] V. Balasubramanian and P. Kraus, Commun. Math. Phys. 208, 413 (1999).

[4] M. Hennigson and K. Skenderis, J. High Energy Phys. 07, 023 (1998); S. Hyun, W. T. Kim, and J. Lee, Phys. Rev. D 59, 084020 (1999).

[5] R. Emparan, C. V. Johnson, and R. C. Myers, Phys. Rev. D 60, 104001 (1999).

[6] R. B. Mann, Phys. Rev. D 60, 104047 (1999); 61, 084013 (2000); S. Das and R. B. Mann, J. High Energy Phys. 08, 033 (2000).

[7] A. M. Awad and C. V. Johnson, Phys. Rev. D 61, 084025 (2000).

[8] M. H. Dehghani and R. B. Mann, Phys. Rev. D 64, 044003 (2001).

[9] A. Strominger, J. High Energy Phys. 10, 034 (2001); 11, 049 (2001); V. Balasubramanian, P. Horova and D. Minic, ibid. 05, 043 (2001); E. Witten, hep-th/0106109; D. Klemm, Nucl. Phys. B625, 295 (2002); V. Balasubramanian, J. D. Boer and D. Minic, Phys. Rev D (to be published), hep-th/0110108; S. Nojiri and S. D. Odintsov, Phys. Lett. B 519, 145 (2001); J. High Energy Phys. 12, 033 (2001); Phys. Lett. B 523, 165 (2001); 528, 169 (2002); T. Shiromizu, D. Ida, T. Torii, J. High Energy Phys. 11, 010 (2001); R. G. Cai, Phys. Lett. B 525, 331 (2002); R. G. Cai, Y. Myung, and Y. Zhang, Phys. Rev. D (to be published); R. Bousso, A. Maloney and A. Strominger, ibid. (to be published); A. M. Ghezelbash and R. B. Mann, J. High Energy Phys. 01, 005 (2002).

[10] M. H. Dehghani, Phys. Rev. D 65, 104003 (2002); (to be published), hep-th/0201128.

[11] D. Klemm, V. Moretti and L. Vanzo, Phys. Rev. D 57, 6127 (1998).

[12] D. Klemm, J. High Energy Phys. 11, 019 (1998).

[13] S. Aminneborg I. Bengtsson, S. Holst and P. Peldan, Class. Quantum Grav. 13, 2707 (1996);

[14] R. B. Mann, Class. Quantum Grav. 14, L109 (1997); L. Vanzo, Phys. Rev. D 56, 6475 (1997).

[15] R. G. Cai, Phys. Rev. D 63, 124018 (2001).

[16] A. M. Ghezelbash D. Ida, R. B. Mann and T. Shiromizu, hep-th/0201004.

[17] J. D. Brown and J. W. York, Phys. Rev. D 47, 1407 (1993).

[18] J. D. Brown, J. Creighton and R. B. Mann, Phys. Rev. D 50, 6394 (1994); K. C. K. Chan, J. D. E. Creighton, and R. B. Mann Phys. Rev. D 54, 3892 (1996).

[19] J. F. Plebanski and M. Demianski, Ann. Phys. (N.Y.) 98, 98 (1976).

[20] S. W. Hawking, C. J. Hunter, and M. M. Taylor-Robinson, Phys. Rev. D 59, 064005 (1999). 